\title{
Modélisation et simulation numérique de l'hydroformage
}

\section{Modeling and numerical simulation of hydroforming}

\author{
Nassraoui Mohammed ${ }^{1}$, Radi Bouchaib ${ }^{2}$ \\ ${ }^{1}$ Laboratoire de Mécanique, Productique et Génie Industriel, EST, Université Hassan II, Maroc, nassraoui@yahoo.fr \\ ${ }^{2}$ Ingénierie Mécanique, Management Industriel et Innovation, FST, Université Hassan I, Maroc, bouchaib.radi@yahoo.fr
}

RÉSUMÉ. Le procédé d'hydroformage est fort de ses avantages technologiques, il n'en demeure pas moins qu'une bonne maîtrise de ce procédé est vitale pour son développement. Dans un contexte industriel de plus en plus compétitif, les constructeurs automobiles sont amenés à réduire les délais de conception et les coûts de développement de leurs nouveaux produits et procédés tel l'hydroformage [RAD 17]. La simulation numérique est devenue aujourd'hui un outil incontournable pour répondre à ces besoins en réduisant le nombre de campagne d'essais, les coûts d'outillage et les délais de conception. La striction, la rupture et le plissement sont les principaux modes de défaillance qui entraînent le rebut systématique des pièces. C'est pourquoi, améliorer la prédiction d'apparition de ces défauts lors de la simulation va dans le sens d'une meilleure maîtrise du procédé. Dans ce travail, on présente une étude théorique et une simulation numérique du procédé et on compare les résultats.

ABSTRACT. The hydroforming process is strong in its technological advantages; it is nonetheless true that a good control of this process is vital for its development. In an increasingly competitive industrial environment, automakers are reducing design time and the cost of developing new products and processes such as hydroforming. The numerical simulation has now become an essential tool to meet these needs by reducing the number of tests, tooling costs and design time. Necking, breaking and wrinkling are the main failure modes that cause the systematic rejection of parts. Therefore, improving the prediction of appearance of these defects during the simulation is in the direction of better control of the process. In this article, we present a theoretical study and a numerical simulation of the process and we compare the results.

MOTS-CLÉS. Hydroformage, loi élastoplastique, simulation numérique, éléments finis.

KEYWORDS. Hydroforming, elastoplastic model, Numerical simulation, finite element.

\section{Introduction}

Le recours à ce procédé est justifié par l'ensemble des avantages qu'il présente par rapport aux procédés classiques tels que l'emboutissage, le pliage ou le soudage. En effet, le procédé ouvre un domaine de formabilité plus large en comparaison avec les procédés conventionnels de mise en forme. Il présente un champ de contraintes résiduelles moins important et gère mieux les problèmes de contact et de frottement grâce à l'utilisation de la pression exercée par le fluide. A travers plusieurs études, les auteurs montrent qu'avec la mise en forme par hydroformage le poids total de l'automobile est réduit et par conséquent la consommation de carburant. L'utilisation du procédé se généralise dans plusieurs secteurs industriels. Actuellement, on s'intéresse à l'hydroformage des matériaux composites qui peut avoir un apport considérable dans l'industrie aéronautique. L'hydroformage est une technique de modélisation des métaux permettant d'obtenir des produits plus solides, plus légers et potentiellement plus complexes. Le principal avantage de l'hydroformage est la flexibilité, puisque cette technique est capable de créer des formes irrégulières à géométrie complexe [ASN 03,LEE 02,OHI 06,YUA 06].

\section{Applications de l'hydroformage}

Les applications principales de l'hydroformage peuvent être trouvées dans les industries : aéronautique, automobile, électrotechnique aussi bien dans la production des composants. C'est le domaine du transport qui utilise particulièrement cette technologie puisque le procédé d'hydroformage permet de réduire le poids tout en assurant une bonne rigidité. Plus précisément, le secteur de 
l'automobile et de l'aéronautique y trouvent un grand intérêt. Les applications dans le domaine de l'automobile comprennent, entre autres, les pièces suivantes : Dans le domaine de l'automobile l'hydroformage permet la production des berceaux de moteurs, les collecteurs d'échappement, les vilebrequins, les arbres à cames, etc [AHM 00]. La figure 1 présente quelques produits obtenus par hydroformage de tubes : traverses de pare-chocs et absorbeur de chocs ; montants ; berceaux de moteur et berceaux arrière ; traverses de pare-brise ; pièces de châssis.

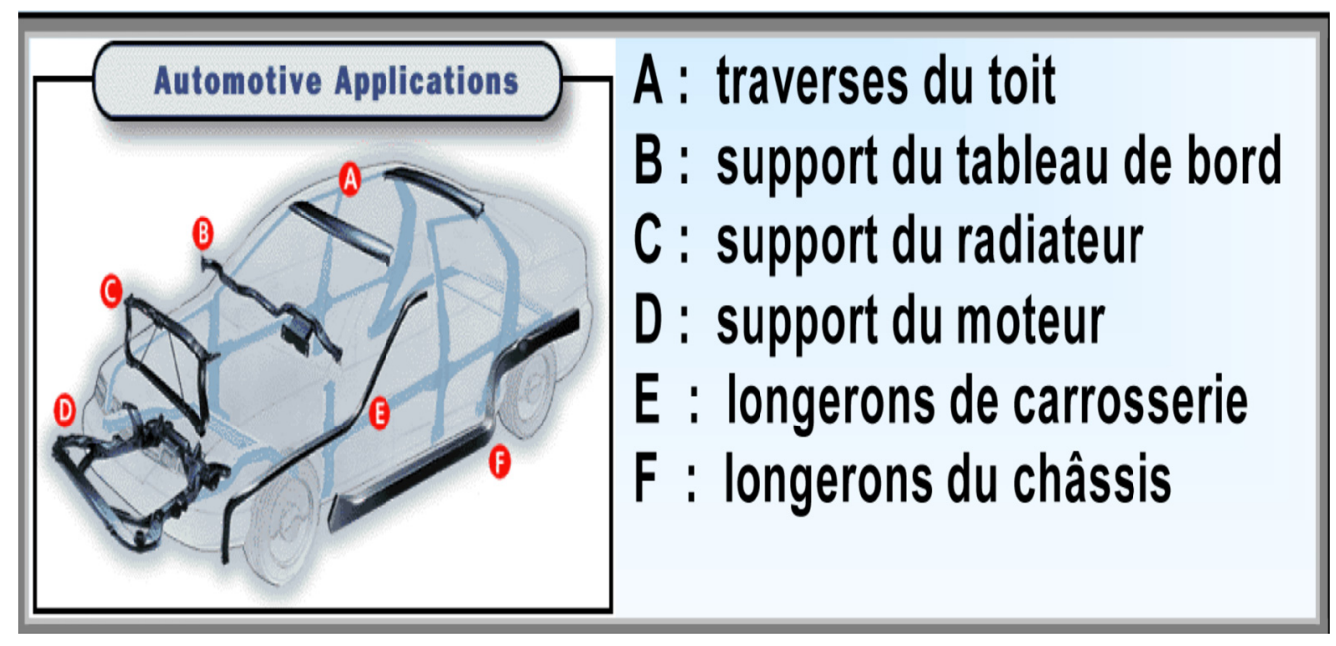

Figure 1. Exemple de produits obtenus par l'hydroformage de tubes (source variform)

\section{Hydroformage de plaques}

La figure 2 présente une méthode typique dans l'hydroformage de feuilles plates, c'est l'emboutissage hydrodynamique (HDD). Le poinçon rigide se déplace vers la chambre fluide et la tôle est forcée de prendre la forme de poinçon sous la pression du fluide variant entre 30 et $150 \mathrm{MPa}$ et pouvant atteindre plus de $200 \mathrm{MPa}$ pour des cas exceptionnels. La pression appliquée sert aussi à éliminer le contact direct entre la feuille métallique et la partie supérieure de la matrice qui réduit l'effet du frottement plaque/matrice. Avec ce procédé, la valeur de la limite du rapport de déformation «limit drawing ratio» (LDR) de la feuille métallique peut être augmentée et la qualité de la pièce améliorée [LAN 04].

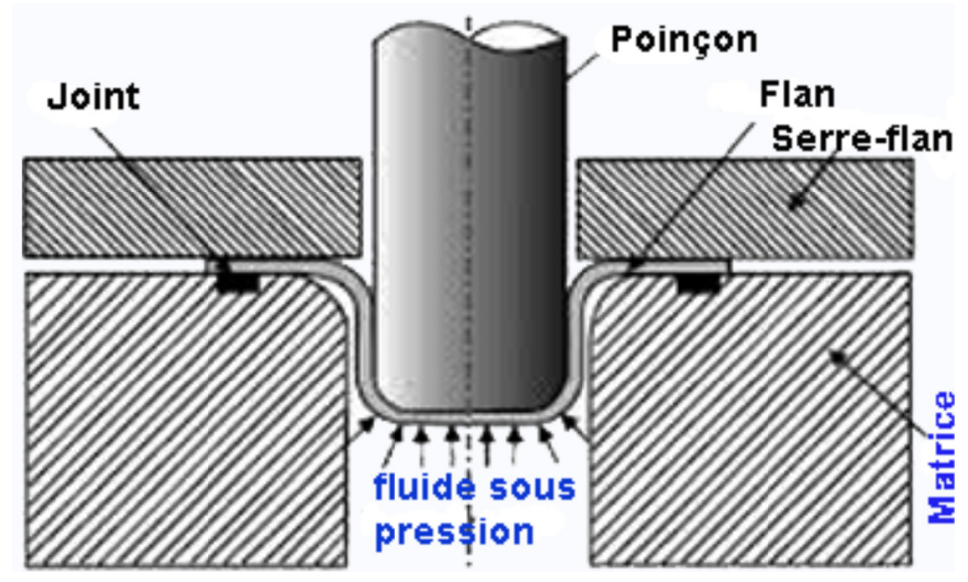

Figure 2. Exemple d'hydroformage de plaques [Che10].

\section{Modélisation de l'hydroformage}

On présente les notions de base relatives à la modélisation du comportement mécanique des structures minces. Nous insistons en particulier sur la formulation théorique permettant de modéliser le comportement mécanique des structures minces sous sollicitations thermo-mécanique en tenant compte des instabilités plastiques. On note que la déformation limite avant rupture des matériaux dépend de 
l'état des contraintes : un état de forte contrainte hydrostatique en compression referme les cavités et ralentit par conséquent l'initiation et la croissance de l'endommagement.

Dans de nombreux procédés de mise en forme travaillant dans le domaine de compression (laminage, extrusion, filage), les déformations sont très élevées dans le matériau sans atteindre la limite de l'endommagement [DEC 09]. Dans le cas des procédés où le matériau travaille plutôt dans le domaine de la traction comme l'emboutissage ou l'hydroformage les déformations sont limitées par les instabilités plastiques.

Pour augmenter la formabilité des matériaux dans les procédés de formage de tôle, la diminution voire la disparition de ces instabilités plastiques est nécessaire. De nombreux mécanismes permettent l'augmentation de la formabilité des tôles minces, on peut citer la vitesse de déformation, la température et l'anisotropie, la microstructure. Pour des applications simples, les lois de Swift ou Voce sont largement utilisées pour reproduire l'écrouissage isotrope lors de trajets de chargement monotones. Tel n'est pas le cas, cependant, lors de changements de trajets de déformation induisant des phénomènes complexes. De plus, dans les processus de mise en forme les grandes déformations irréversibles peuvent engendrer des mécanismes d'endommagement conduisant à la localisation de la déformation qui précède la rupture.

On se restreint au cadre de sollicitations non couplées à la température ou à la vitesse de déformation. En revanche, la modélisation prend bien en compte les effets d'anisotropie et se place dans le cadre des grandes déformations. Le formalisme général de lois de comportement dans le cadre des matériaux standard généralisés offre un cadre systématique de modélisation et assure la satisfaction des deux principes de la thermodynamique. On considère donc le potential d'état (énergie libre de Helmholtz), par la donnée d'une fonction scalaire convexe contenant l'origine d'une partie élastique et plastique :

$$
\Psi=\Psi_{e}\left(\varepsilon^{e}\right)+\Psi_{p}(r, \alpha)
$$

La relation contrainte déformation est donnée par la relation connue sous le nom de la loi de Hooke généralisée par l'expression :

$$
\varepsilon_{i j}^{e}=\frac{1+v}{E} \sigma_{i j}-\frac{v}{E} \Sigma \delta_{i j}
$$

avec $E$ le module de Young et $v$ le coefficient de Poisson. Le critère de plasticité de Von-Mises permet de déterminer le seuil d'écoulement plastique d'un matériau métallique isotrope. Le matériau est supposé entrer en plasticité lorsque son énergie élastique de cisaillement atteint une valeur seuil. Pour un tenseur des contraintes diagonales, le critère de Von-Mises s'écrit [Aza07] :

$$
\sigma_{e q}=\sqrt{\frac{1}{2}\left[\left(\sigma_{1}-\sigma_{2}\right)^{2}+\left(\sigma_{2}-\sigma_{3}\right)^{2}+\left(\sigma_{3}-\sigma_{1}\right)^{2}\right]}
$$

\subsection{Loi élasto-plastique}

Le comportement élasto-plastique repose sur une décomposition de la déformation totale apparente en une partie élastique réversible et une partie plastique irréversible. Lorsque la partie élastique est suffisamment faible, il est courant d'adopter une décomposition additive du tenseur taux des déformtions :

$$
\dot{\varepsilon}=\dot{\varepsilon}^{\mathrm{e}}+\dot{\varepsilon}^{p}
$$

$\dot{\varepsilon}^{\mathrm{e}}$ et $\dot{\varepsilon}^{p}$ sont les tenseurs taux de déformation, respectivement, élastique et plastique. Le tenseur taux des déformations total $\dot{\varepsilon}$ correspond, dans l'hypothèse des petites déformations, à la partie symétrique du tenseur gradient du champ de vitesse $\mathrm{V}$, qui s'écrit : 


$$
\dot{\varepsilon}=\frac{1}{2}\left[\left(\operatorname{grad}(V)+\operatorname{grad}(v)^{\mathrm{T}}\right)\right]
$$

L'étude du comportement des tôles est le plus souvent abordée dans le cadre d'une approche élastoplastique pour la plupart des procédés de mise en forme de tôles. La théorie élastoplastique comporte elle-même deux approches différentes décrivant chacune d'elle une échelle physique du comportement [BEN 11].

L'essai de traction suivant la direction de laminage est souvent choisi comme essai de référence pour établir la courbe d'écrouissage reliant l'évolution de la limite élastique à la variable interne d'écrouissage. On approche ces courbes par des fonctions analytiques qui peuvent prendre plusieurs formes. On considère la loi de Swift :

$$
\sigma=k\left(\varepsilon_{0}+\varepsilon\right)^{n}
$$

\subsection{Essai de gonflage circulaire}

L'essai de gonflage hydraulique circulaire est un essai de caractérisation dont l'exploitation peut être double : d'une part il offre la possibilité d'identifier une courbe d'écrouissage qui couvre une grande plage de déformations, et d'autre part, il peut servir à déterminer le point limite en déformation équibiaxiale. Il est bien établi que l'essai de traction, classiquement utilisé dans la caractérisation des tôles, est limité par l'apparition de la striction diffuse qui se manifeste pour une déformation effective de l'ordre du coefficient d'écrouissage. Cependant, en gonflage, la striction apparaît plus « tardivement » et la phase de déformation homogène est alors prolongée. C'est au vu de ces propriétés que notre intérêt s'est porté sur l'utilisation de l'essai de gonflage pour l'identification des courbes d'écrouissage en grandes déformations. L'étude de cet essai a fait l'objet de plusieurs travaux dans la littérature [HIL 50, HIL 80, STO 83, WAN 69], et son domaine d'exploitation n'est pas restreint à l'unique usage en mise en forme des tôles mais il est étendu à d'autres applications telles que la caractérisation de films minces en polymères [ZHE 03], ou de films minces métalliques [LIC 03, PAV 94]. Ces dernières années, l'essai de gonflage a connu un important regain d'intérêt [BAN 01, NOV 01, REE 99], qui peut être attribué à l'élargissement et à l'industrialisation du procédé d'hydroformage, procédé qui s'apparente beaucoup aux essais de gonflage hydraulique.

\subsection{Solution analytique}

On se propose dans ce qui suit d'analyser l'essai de gonflage circulaire tout en adoptant le critère de plasticité anisotrope de Hill48 pour la description du comportement des tôles. Pour une loi d'écoulement associée, la déformation équivalente s'écrit : Critère de Hill48 en contraintes planes : avant d'aborder le dépouillement de l'essai, on explicite d'abord une expression du critère de Hill (1948) sous l'hypothèse des contraintes planes et en supposant le repère principal des contraintes coïncidant avec le repère d'orthotropie. La contrainte équivalente est alors définie dans le repère principal par [BEN 05] :

$$
\bar{\sigma}=\frac{\sqrt{r_{90}\left(1+r_{0}\right)-2 r_{0} r_{90} \Omega+r_{0}\left(1+r_{90}\right) \Omega^{2}}}{\sqrt{r_{90}\left(1+r_{0}\right)}} \sigma_{1}
$$

avec $\Omega$ défini par :

$$
\Omega=\frac{\sigma_{2}}{\sigma_{1}}
$$

Pour une loi d'écoulement associée, la déformation équivalente s'écrit :

$$
d \bar{\varepsilon}=\frac{\sqrt{1+r_{0} \sqrt{r_{0}\left(1+r_{90}\right)+2 r_{0} r_{90} \beta+r_{90}\left(1+r_{0}\right) \beta^{2}}}}{\sqrt{r_{0}+r_{0} r_{90}+r_{0}^{2}}} d \varepsilon_{1}
$$


avec $\beta$ défini par :

$$
\beta=\frac{d \varepsilon_{2}^{p}}{d \varepsilon_{1}^{p}}
$$

Par ailleurs, il est possible de démontrer, en utilisant l'équation 10 et la loi de la normalité, que le rapport des contraintes est relié au rapport des déformations par la relation suivante :

$$
\beta=\frac{r_{0}\left(1+r_{90}\right) \Omega-r_{0} r_{90}}{r_{90}\left(1+r_{0}\right)-r_{0} r_{90} \Omega}
$$

Equation d'équilibre : Dans la suite, on se place dans le repère d'orthotropie du matériau. Dans ce repère, l'état de contrainte au pôle d'une tôle gonflée est défini par le tenseur des contraintes suivant :

$$
\sigma=\left[\begin{array}{ccc}
\sigma_{1} & 0 & 0 \\
0 & \sigma_{2} & 0 \\
0 & 0 & 0
\end{array}\right]
$$

Comme les contraintes principales sont confondues avec les axes d'orthotropie, la condition de coaxialité est par conséquent vérifiée. Il s'en suit que le tenseur des déformations est aussi diagonal dans le repère d'orthotropie et s'écrit :

$$
\varepsilon=\left[\begin{array}{ccc}
\varepsilon_{1} & 0 & 0 \\
0 & \varepsilon_{2} & 0 \\
0 & 0 & \varepsilon_{3}
\end{array}\right]
$$

L'équation d'équilibre, ou équation de Pascal, est définie par :

$$
\frac{\sigma_{1}}{R_{\theta}}+\frac{\sigma_{2}}{R_{\phi}}=\frac{P}{e}
$$

où $\sigma_{1}, \sigma_{2}$ sont les contraintes principales (confondues avec les axes du matériau), $R_{\theta}$ et $R_{\phi}$ sont les rayons de courbure respectivement suivant les deux directions principales, $\mathrm{P}$ la pression de gonflage et e l'épaisseur courante au pôle de la tôle. En introduisant le rapport des contraintes $\Omega$ et en supposant que $R_{\theta}=R_{\phi}=\rho$, l'équation d'équilibre se réduit à :

$$
\sigma_{1}=\frac{P . \rho}{((1+\Omega) \cdot e)}
$$

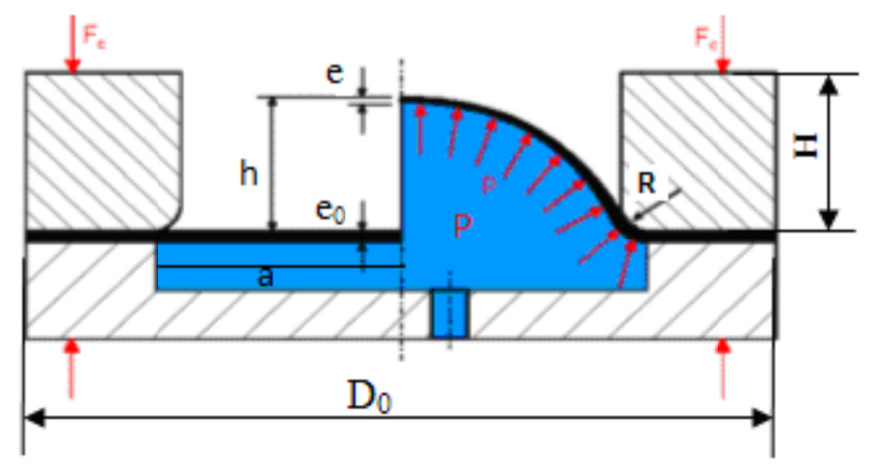

Figure 3. Essai de gonflage circulaire

En supposant que la géométrie de la tôle déformée est de forme sphérique, le rayon de courbure $\rho$ peut être déduit à partir de la hauteur du dôme $h$ sur un rayon a selon la relation du sphéromètre :

$$
\rho=\frac{a^{2}+h^{2}}{2 h}
$$


On peut démontrer aussi que la déformation principale est définie par :

$$
\varepsilon_{1}=\ln \left(1+\frac{h^{2}}{a^{2}}\right)
$$

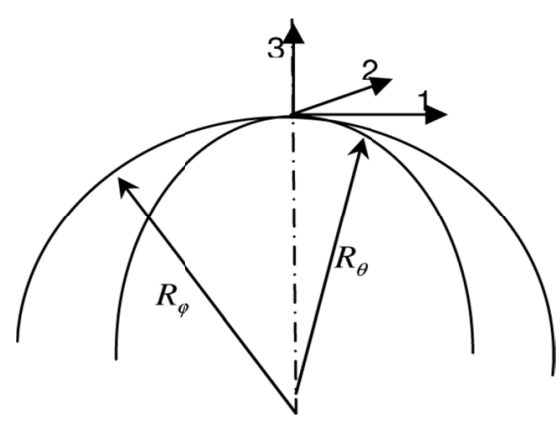

Figure 4. Géométrie de la déformée [BEN 05].

A ce stade, on connaît la contrainte et la déformation principale en fonction des variables mesurables $(h, a, \rho, P)$. Il reste donc à déterminer le rapport de contrainte $\Omega$ pour pouvoir établir une relation directe entre les efforts et les déformations. Pour se faire, on va supposer en première approximation un état d'anisotropie normale. Ceci réduit le nombre des paramètres d'anisotropie au seul paramètre $r$. Dans ce cas, le comportement est isotrope dans le plan de la tôle, il en découle donc un état de contrainte et de déformation équi-biaxiale $(\beta=\Omega=1)$. La contrainte et la déformation équivalente sont alors définies par :

$$
\bar{\sigma}=k\left(\sqrt{\frac{2}{(1+\bar{r})}} \sigma_{1}\right), \quad d \bar{\varepsilon}=\sqrt{2(1+\bar{r})} d \varepsilon_{1}
$$

En utilisant une loi d'écrouissage de type Hollomon :

$$
\bar{\sigma}=k \bar{\varepsilon}^{n}
$$

combinée avec les équations [7] et [18] et en tenant compte de l'expression de la déformation équivalente, la contrainte équivalente est alors définie par :

$$
\bar{\sigma}=k\left(\sqrt{2(1+\bar{r})} \ln \left(1+\frac{h^{2}}{a^{2}}\right)\right)^{n}
$$

En se basant sur l'hypothèse d'incompressibilité plastique, l'épaisseur courante au pôle est définie à partir de l'épaisseur initiale $\mathrm{e}_{0}$ par :

$$
e=e_{0}\left(1+\frac{h^{2}}{a^{2}}\right)^{2}
$$

L'équation finale reliant la pression de gonflage à la hauteur du dôme est déduite à partir des équations et tenant compte de l'expression de la contrainte équivalente :

$$
P=2 \sqrt{2(1+\bar{r})} K \frac{e_{0} h}{a^{2}} \frac{1}{\left(\left(1+\frac{h^{2}}{a^{2}}\right)\right)^{3}}\left(\sqrt{2(1+\bar{r})} \ln \left(1+\frac{h^{2}}{a^{2}}\right)\right)^{n}
$$

Sur la figure 5 sont reportées les courbes d'évolution de la pression de gonflage en fonction de la hauteur du dôme pour divers coefficients d'anisotropie. Il convient de souligner les remarques suivantes : 
- la pression de gonflage augmente graduellement jusqu'à atteindre le maximum de pression, appelée pression de rupture, au-delà duquel commence une phase de déformation dite instable marquée par la décroissance de la pression bien que la tôle continue à gonfler.

- d'après la prédiction du modèle, la pression de rupture Pmax est très sensible au coefficient d'anisotropie ; $P_{\max }$ augmente avec $\bar{r}$. En même temps, le modèle montre que la hauteur qu'atteint le matériau à la pression de rupture est indépendante du coefficient d'anisotropie.

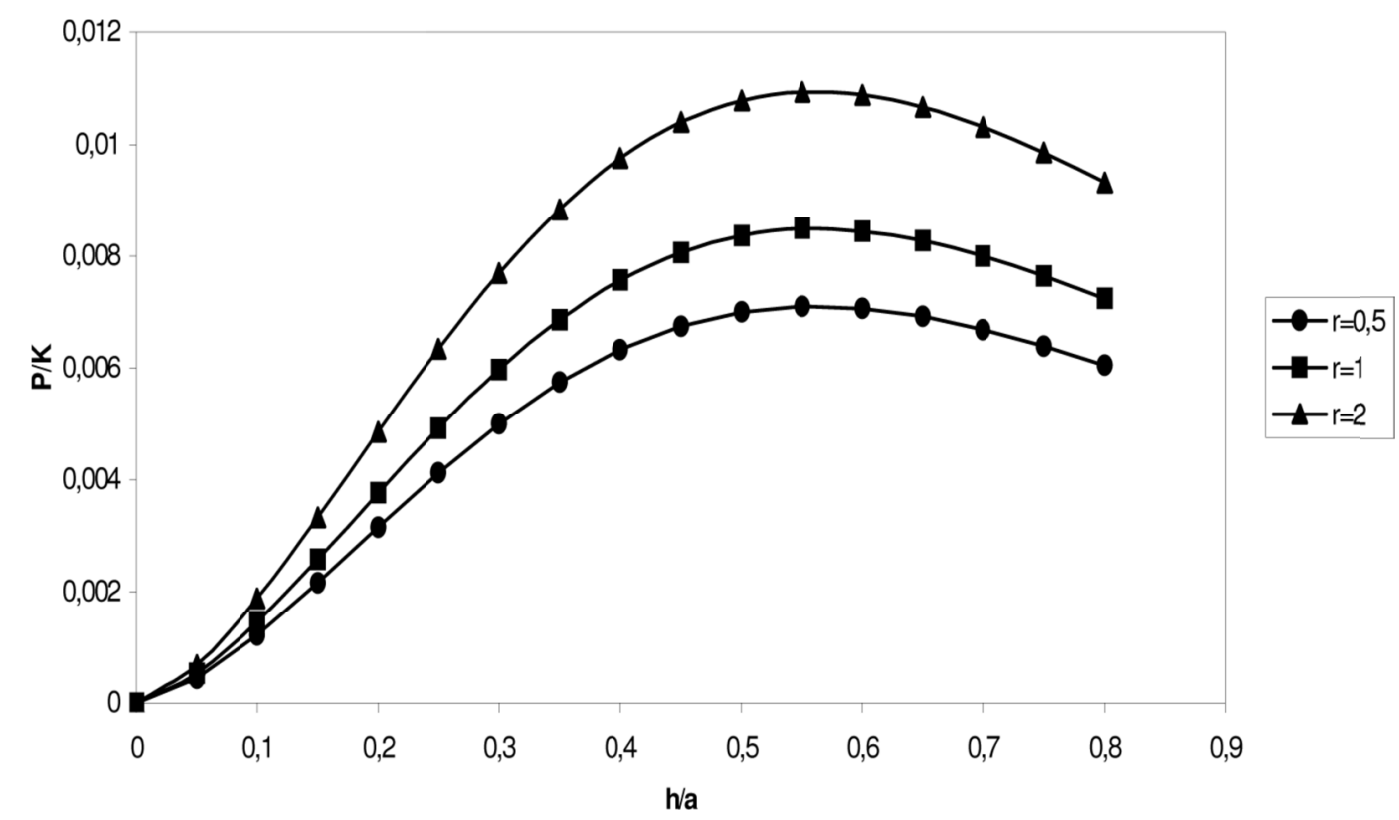

Figure 5. Variation de la hauteur en fonction de la pression et du coefficient d'anisotropie [Ben05].

\subsubsection{Application numérique}

Pour quantifier la valeur de la hauteur au pôle en fonction de la pression appliquée sur la tôle $\mathrm{N}^{\circ} 2$ en utilisant l'équation [22], on prend les valeurs données dans le tableau suivant 1 avec:

- Coefficient d'anisotropie $\bar{r}$;

- Rayon a de la tôle en contact avec le fluide ; $(\mathrm{a}=\mathrm{D} / 2=120=2=60 \mathrm{~mm})$;

- Coefficient K;

- Coefficient d'écrouissage n ;

- Pression du fluide P.

\begin{tabular}{|c|c|c|c|c|}
\hline$\overline{\boldsymbol{r}}$ & $\boldsymbol{a}(\mathbf{m m})$ & $\boldsymbol{K}(\mathbf{M P a})$ & $\boldsymbol{n}$ & $\boldsymbol{P}(\mathbf{M P a})$ \\
\hline 1,68 & 60 & 536 & 2,24 & 2,061 \\
\hline
\end{tabular}

Tableau 1. Tableau des données

En utilisant la formule de l'équation [22], on trouve la hauteur $\mathrm{h} \approx 10,8 \mathrm{~mm}$.

\section{Simulation numérique}

Dans cette section, on va montrer l'influence des paramètres d'hydroformage des tôles en utilisant le code de calcul ANSYSV13(3D) qui permet de modéliser les différentes structures présentant des formes géométriques complexes. Les modèles géométriques appliqués constituent un ensemble de 
singularités ayant des formes géométriques de base. Pour cela nous avons choisis différents paramètres d'hydroformage des tôles et nous avons essayé de voir quel est le déplacement maximum pour chaque cas étudié. Afin d'étudier l'influence de différents paramètres intrinsèques à la modélisation éléments finis, plusieurs modèles ont été élaborés. Les différentes études menées ont permis de tester l'influence :

- de l'épaisseur de la tôle et la pression du fluide (épaisseur de 1 à $3 \mathrm{~mm}$ la pression de 0,2 à 2 $\mathrm{MPa})$;

- du coefficient de frottement $\mu$, variant de 0 à 0,2 ;

- du type d'élément (C3D4 1, C3D8 2 et C3D20 3);

- du nombre d'éléments dans l'épaisseur, la largeur et la circonférence de lla tôle, ou variant ;

- de 23292 à 113250 éléments de type C3D4 ;

- du maillage afin de gagner le temps de calcul.

Les modèles éléments finis sont comparés entre eux afin de mettre en évidence les paramètres influents les simulations numériques. Cette comparaison permet aussi de choisir le modèle éléments finis le plus performant afin de le comparer aux résultats expérimentaux préliminaires réalisés sur l'acier doux. Le comportement mécanique du matériau a été simulé à température ambiante et à faible vitesse de déformation par le logiciel. Les caractéristiques élastiques et géométriques des tôles ainsi que la masse volumique sont rassemblées dans les tableaux suivants (tableaux 2 et 3 ) :

\begin{tabular}{|c|c|c|c|}
\hline Désignation & $\boldsymbol{\rho}\left(\mathbf{k g} \cdot \mathbf{m}^{-\mathbf{3}}\right)$ & $\mathbf{E}(\mathbf{G P a})$ & $\boldsymbol{v}$ \\
\hline acier doux $(\mathrm{E} 24)$ & 7800 & 204 & 0,3 \\
\hline
\end{tabular}

Tableau 2. Caractéristiques élastiques et masse volumique du matériau étudié

\begin{tabular}{|c|c|c|c|}
\hline$\varnothing$ tôle(mm) & e tôle(mm) & $\varnothing$ matrice(mm) & rayon de courbure matrice(mm) \\
\hline 204,3 & 2 & 120 & 5 \\
\hline
\end{tabular}

Tableau 3. Caractéristiques de la tôle $N^{\circ} 2$

La simulation numérique des essais d'hydroformage portera sur les mêmes caractéristiques des tôles utilisées lors des essais expérimentaux. Afin de bien modéliser ces essais nous avons utilisé le code de calcul ANSYS V13(3D) pour son environnement et sa puissance de calcul.

Le montage des pièces mécaniques a été réalisé sur un logiciel de DAO et ensuite transféré vers le code Ansys V13 (voir figure 6).

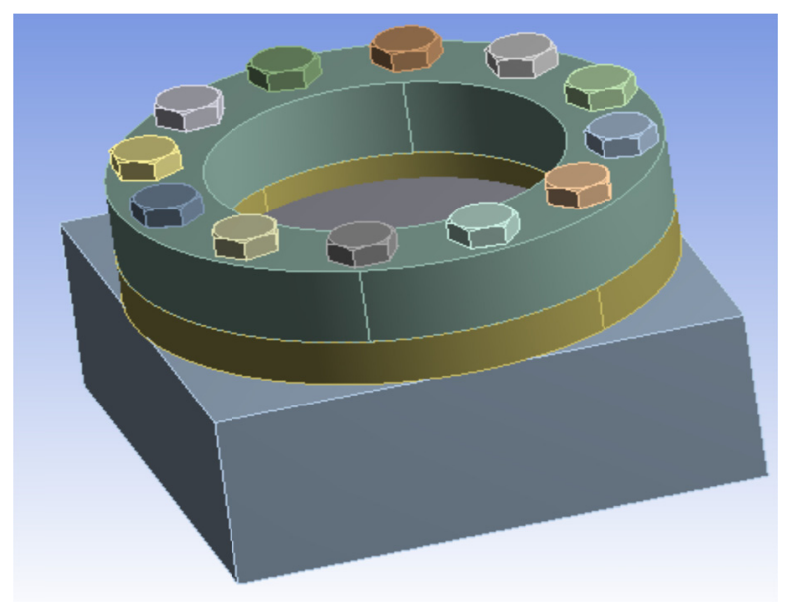

Figure 6. Dessin 3D du montage d'essai 


\subsection{Simulation du problème avec ANSYS}

Le code d'éléments finis ANSYS V13 (3D) est utilisé dans cette partie pour simuler le comportement du mécanisme de contact par frottement ou sans frottement des deux corps (matrice et tôle) lors des essais. Ce code possède des algorithmes de gestion du contact avec frottement basés sur la méthode des multiplicateurs de Lagrange, ou la méthode de pénalisation. Les simulations présentées dans notre étude considèrent le contact sans frottement. L'application de la pression sur la tôle est simulée comment une pression uniforme durant l'essai. le matériau choisi de la tôle a un comportement élastique isotrope dont les caractéristiques mécaniques sont récapitulées dans le tableau suivant. Les caractéristiques de conception des pièces sont également fournies directement par le code Ansys V13 ; données dans le tableau 4.

\begin{tabular}{|c|c|c|c|c|c|c|}
\hline Volume & Masse & Eléments & Nouds & Ip1 kg.mm & ${\text { Ip2 } \mathbf{~ k g} \cdot \mathbf{m m}^{2}}^{\mathbf{2}}$ & ${\text { Ip3 } \mathbf{~ k g} \cdot \mathbf{m m}^{\mathbf{2}}}$ \\
\hline 7,79 & 21,93 & 399 & 20400 & 0,3 & 536 & 0,24 \\
\hline
\end{tabular}

Tableau 4. Caractéristiques de conception de la tôle $N^{\circ} 2$

Le coefficient de frottement $\mu$ est égal à 0,2 au niveau de la zone de contact. Dans le cas avec frottement, cette dernière se situe de l'interface de contact, les contraintes de cisaillement provoquées par le frottement à ce niveau-là sont à l'origine de ce phénomène. Le coefficient de frottement dépend de beaucoup de paramètres (pression, vitesse de glissement, température, humidité, etc.). Nous rappelons que l'ANSYS peut, au choix, utiliser une méthode de multiplicateurs de Lagrange ou bien une méthode de Lagrangien augmenté, une méthode de pénalisation pour résoudre le problème de contact. C'est cette dernière qui a été sélectionnée dans ce travail.

Pour commencer l'étude, on a créé une structure sur ANSYS Wb V13 qui représente le montage tôle et matrice. Puis, on a fait le maillage et on a défini les conditions aux limites pour le mettre ensuite sur l'ANSYS Multiphysics et initialiser le calcul.

Afin d'économiser en temps de calcul, le modèle éléments finis maillé avec les paramètres stabilisés (totalement raffiné) a été comparé à un modèle éléments finis avec un maillage raffiné uniquement sur une portion de la pièce. La taille d'éléments dans la zone raffinée est identique au modèle totalement raffiné. Une fois la structure est créée, on a fait le maillage. Il s'agit d'un maillage progressif. Le modèle tôle-matrice a été maillé par des éléments volumiques tétraédriques et quadratiques à 10 nœuds (figure 7).

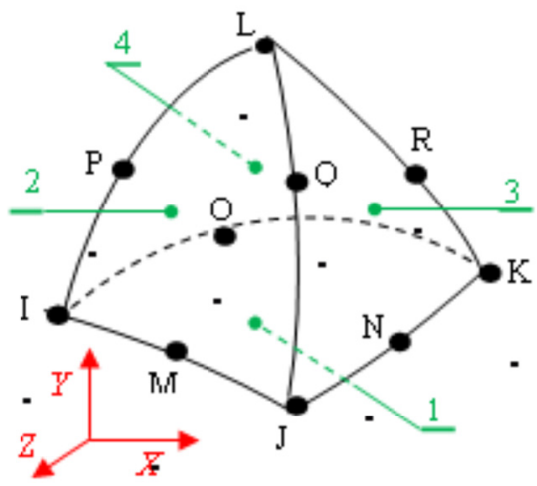

Les nouds: I, J, K, L, M, N, O, P, Q, R

Les faces: $1(\mathrm{~J}-\mathrm{I}-\mathrm{K}), 2(\mathrm{I}-\mathrm{J}-\mathrm{L}), 3(\mathrm{~J}-\mathrm{K}-\mathrm{L}), 4(\mathrm{~K}-\mathrm{I}-\mathrm{L})$

Figure 7. Elément tétraèdre quadratique isoparamétrique à 10 nœuds 
L'ANSYS Workbench effectue un maillage automatique (par défaut). Ensuite on a essayé de raffiner le maillage de la tôle en intervenant dans le changement des paramètres choisis par défaut. Les figures 7 et 8 donnent respectivement un maillage volumique de la tôle.

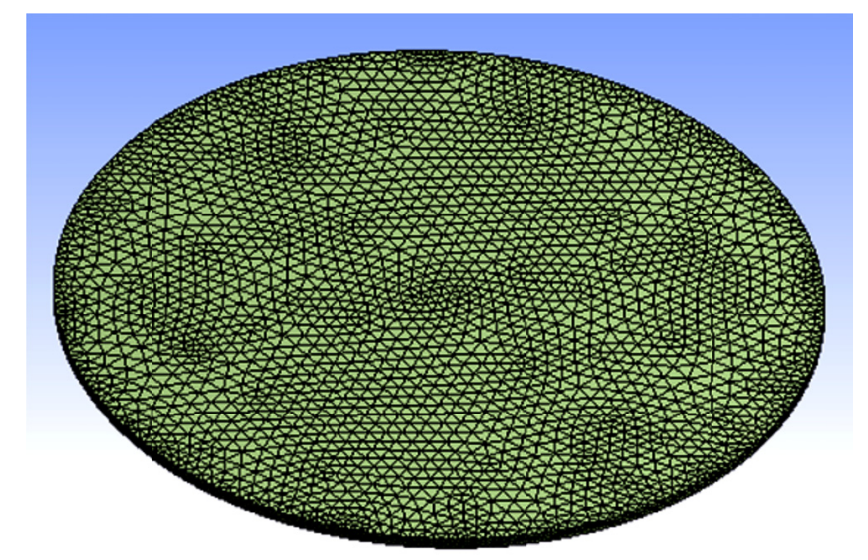

Figure 8. Maillage de la tôle Nœuds éléments

Pour appliquer le modèle mécanique d'élément finis avec le montage, nous considérons les hypothèses suivantes :

- La pression appliquée est uniformément répartie sur la zone de contact de la tôle.

- Le contact entre matrice et tôle est considéré sans frottement.

- Les matériaux des pièces de montage sont homogènes et leurs propriétés sont invariables avec la température.

Afin d'économiser en temps de calcul, le modèle éléments finis maillé avec les paramètres stabilisés (totalement raffiné) a été comparé à un modèle éléments finis avec un maillage raffiné uniquement sur une portion de la pièce. La taille d'éléments dans la zone raffinée est identique au modèle totalement raffiné.

Les différentes conditions aux limites imposées au modèle éléments finis de la tôle et de la matrice en configurations encastrées, compte tenu de son environnement direct, sont les suivantes :

- La tôle est bloquée aux extrémités de la circonference par le montage qui permet de la maintenir.

- La matrice est encastrée par 12 perçages au niveau du bol de fixation qui permettent de la maintenir fixe par rapport au socle.

- Une pression $\mathrm{P}$ variant de 0,2 MPa à $2 \mathrm{MPa}$ selon le diamètre de la tôle et son épaisseur.

- Pour l'étude de contact entre la matrice et la tôle, nous avons pris la méthode de pénalité.

- Pour la gestion du contact plutôt que l'utilisation des multiplicateurs de Lagrange. Plusieurs arguments motivant ce choix en premier lieu, c'est une méthode simple à adapter et elle ne génère pas d'inconnues supplémentaires. D'autre part, les mesures considérées de compression de la tôle sur un support rigide montrent un comportement non-linéaire, principalement dû aux aspérités des surfaces de contact et/ou au comportement élastique non-linéaire du matériau de tôle (figure 9). 


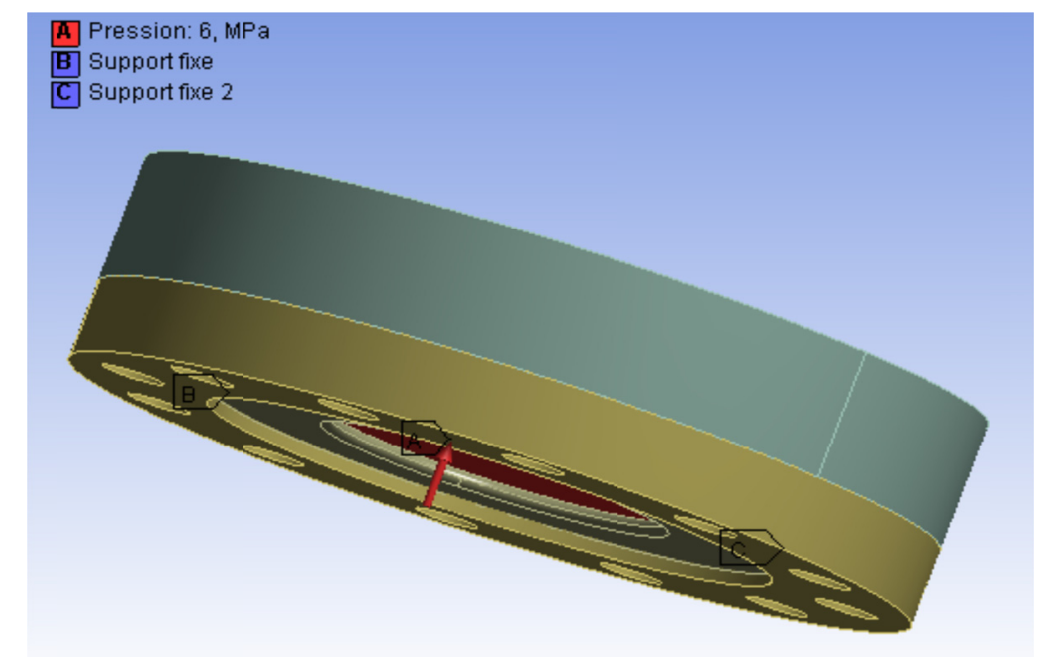

Figure 9. Conditions aux limites et chargement imposés

Une fois les données sont introduites, on lance la résolution pour les différents essais. Le choix d'un résultat parmi tant d'autres obtenus se fait selon le besoin de l'étude à effectuer. Une fois les résultats obtenus, il ne reste qu'à varier certains paramètres (caractéristiques physiques) par rapport à certains autres afin de déterminer les plus influents.

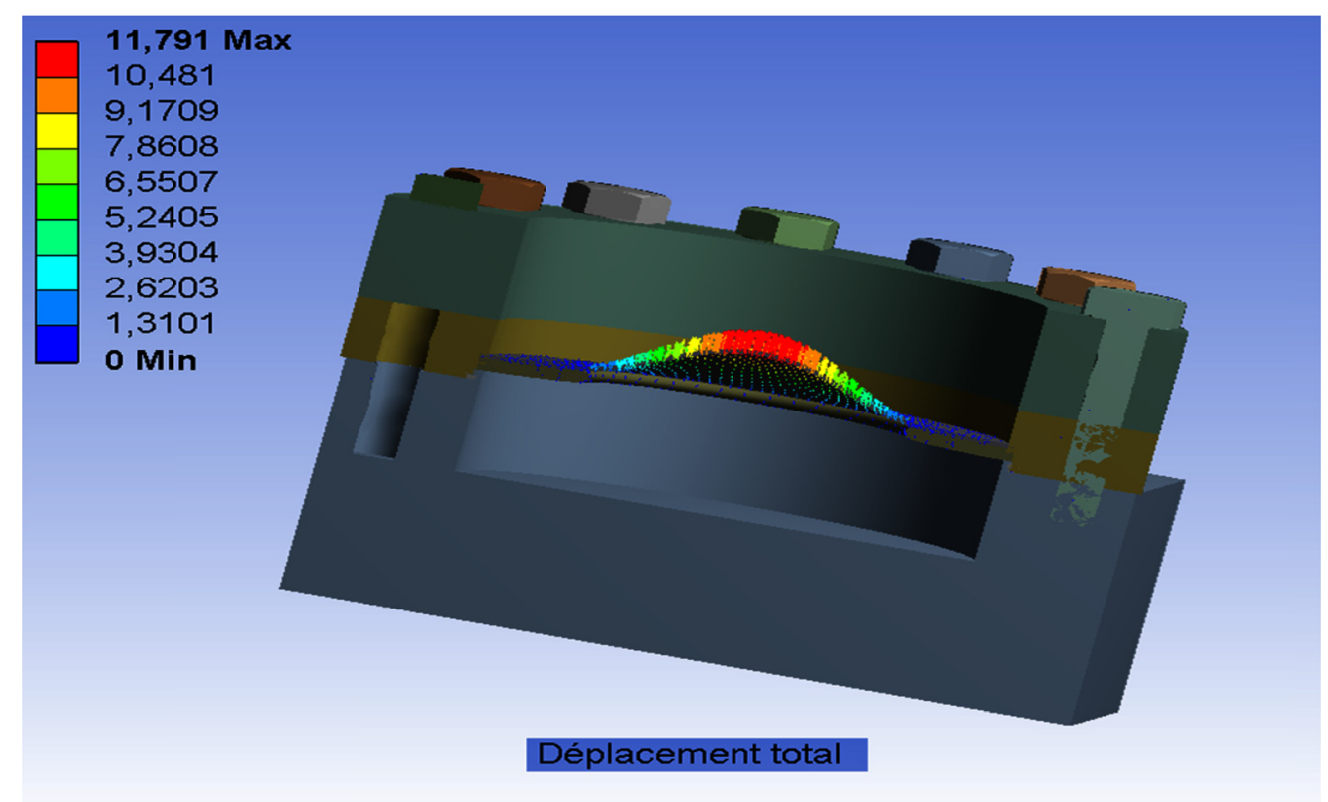

Figure 9. Déformée de la tôle

\subsection{Résultats numériques}

La méthode des éléments finis est utilisée pour explorer la contrainte et le déplacement ainsi que l'influence de l'écrouissage, de l'anisotropie et de la sensibilité de ces paramètres sur la réponse de la tôle. Un modèle tridimensionnel discrétisé en éléments finis (3D) ensuite résolu avec Ansys v13. Il s'agit d'un assemblage tôle et matrice de dimensions et paramètres initiaux. Un profil de pression surfacique en MPa au cours du temps est appliqué à la surface de tôle. La tôle est gonflée sous ce chargement en libérant uniquement ses extrémités dans la direction de l'axe de symétrie de la tôle. Le comportement est formulé dans le cadre des modèles élastoplastiques anisotropes et avec écrouissage isotrope (figure 9). 
6.2.1. Simulation de la tôle $N^{\circ} 2$

\section{Contact entre tôle et matrice}

- type de contact : sans frottement et avec frottement $(\mu=0,2)$

- comportement : symétrique

- formulation : méthode par pénalité

\section{Maillage}

- méthode : balayage

- nœuds intermédiaire d'éléments : supprimé

- sélection source/cible : à parois fixes

- type de maillage face libre : quadrilatères/triangles

- options d'éléments : coque

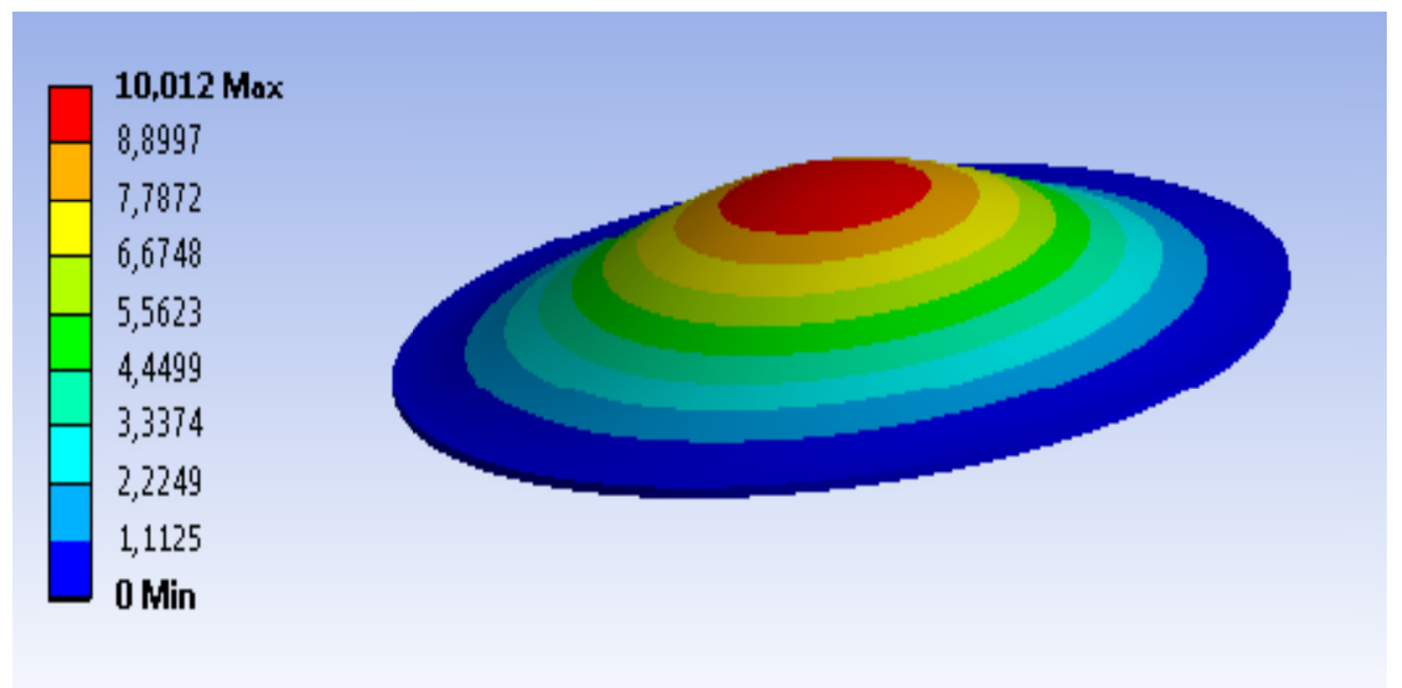

Figure 10. Le déplacement au pôle

Le tableau 5 nous donne les résultats des essais comparés avec les résultats expérimentaux [NAS 18].

\begin{tabular}{|c|c|c|c|c|}
\hline $\begin{array}{c}\text { Pression } \\
\mathbf{( M P a})\end{array}$ & $\begin{array}{c}\text { Dép. simulé avec } \\
\boldsymbol{\mu}=\mathbf{0 , 2}(\mathbf{m m})\end{array}$ & $\begin{array}{c}\text { Dép. simulé sans } \\
\text { frottement } \mathbf{( m m )}\end{array}$ & $\begin{array}{c}\text { Dép. théorique } \\
\mathbf{( m m})\end{array}$ & $\begin{array}{c}\text { Dép. expérimental } \\
\mathbf{( m m})\end{array}$ \\
\hline 2,061 & 9,972 & 10,012 & 10,8 & 10,9 \\
\hline
\end{tabular}

Tableau 5. Comparaison des résultats du déplacement au pôle

\section{Conclusion}

Nous avons proposé un modèle numérique pour simuler le procédé d'hydroformage. Les résultats numériques ont montré la faible influence du frottement entre la matrice et la tôle. Le choix de l'élément fini adéquat sous le code de calcul Ansys en 3D a montré son efficacité pour calculer le déplacement au pôle pour la tôle de $2 \mathrm{~mm}$ d'épaisseur. 


\section{La bibliographie}

[AHM 00] M. Ahmetoglu and T. Altan, «Tube hydroforming state-of-the-art and future trends ». Journal of Materials Processing Technology, 98 :25-33, 2000.

[AGA 08] P. Agati, F. Lerouge, and M. Rossetto, Résistance des matériaux. 2ème Edition, 2008.

[ASN 03] N. Asnafi, T. Nilsson, and G. Lassi, « Tubular hydroforming of automotive side members with extruded aluminium profiles », Journal of materials processing technology, 142 :93-101, 2003.

[AZA 07] M. Azaouzi « Modélisation et optimisation numérique de l'emboutissage de pièces de précision en tôlerie fine», PhD thesis, INPL - Nancy, Nancy - France, 2007.

[BAN 01] D. Banabic, D. Balan, and S. Comsa, « Closed-form solution for bulging through elliptical dies. » Mat. Proc. Tech, $115: 83-86,2001$.

[BEN 05] M. Ben Tahar, « Contribution à l'étude et la simulation du procédé d'hydroformage. » PhD thesis, Ecole des mines de Paris, Paris - France, 2005.

[BEN 11] M.A. Ben Abdessalem, « Optimisation avec prise en compte des incertitudes dans la mise en forme par hydroformage », PhD thesis, INSA Rouen, Rouen - France, 2011.

[CHE 10] M.S. Chebbah, "Simulation et optimisation rapides des paramètres du procédé d'hydroformage de tubes par éléments finis en utilisant une méthode inverse ». PhD thesis, Université de Batna, Batna- Algérie, 2010.

[DEC 09] N. Decultot, « Formage incrémental de tôle d'aluminium : étude du procédé à l'aide de la mesure de champs et identification de modèles de comportement ». PhD thesis, Université -Toulouse, Toulouse - France, 2009.

[HEI 99] P. Hein and F. Vollertsen, « Hydroforming of sheet metal pairs », Journal of Materials Processing Technology, $87: 154-164,1999$.

[HIL 50] R. Hill, « A theory of the plastic bulging of a metal diaphragm by lateral pressure. » Phil. Mag, $7: 1133-1142$, 1950.

[HIL 80] R. Hill and B. Storakers, « Plasticity and creep of pressurized membranes: a new look at the small-deflection theory. » J. Mech. Phys. Solids, $28: 27-48,1980$.

[KAH 10] H. Kahoul, « Modélisation du comportement à la déformation élastique des tôles de construction automobile : influence des singularités », Master’s thesis, Université Mentouri, Constantine - Algérie, 2010.

[LAN 04] L.H. Lang, Z.R.Wang, D.C. Kang, S.J. Yuan, J. Zhang, S.H.and Danckert, and K.B. «Nielsen.Hydroforming highlights : sheet hydroforming and tube hydroforming » Journal of Materials Processing Technology, $151: 165-177$, 2004.

[LEE 02] M.H. Lee, S.M. Sohn, C.Y. Kang, and S.Y. Lee « Study on the hydroforming process for automobile radiator support members». Journal of Materials Processing Technology, 130-131 :115-120, 2002.

[LIC 03] Y. Li and M.J. Cima, « Bulge test on free standing gold thin films», MRS Fall Meeting (Boston) :437-442, Dec 2003.

[NAS 18] M. Nassraoui, Z. Elmaskaoui, B. Radi, «Caractérisation expérimentale de l'hydroformage », à paraitre dans Incertitudes et fiabilité des systèmes multiphysiques, 2018.

[NOV 01] S. Novotny and P. Hein, « Hydroforming of sheet metal pairs from aluminum alloys », Mat. Proc. Tech, 115 :65-69, 2001.

[OHI 06] S.I. Oh, B. H. Jeon, H. Y. Kim, and J. B. Yang, « Applications of hydroforming processes to automobile parts », Journal of Materials Processing Technology, 174 :42-55, 2006.

[PAV 94] V. Paviot, J. MandVlassak, and W.D. JandNix, « Measuring the mechanical properties of thin metal films by means of bulge testing of micromachined windows ", In Stresses and Mechanical Properties V, pages 579-584, Boston USA, 28 Nov-2 Dec 1994.

[RAD 17] B. Radi et A. El Hami, Mise en forme des matériaux : emboutissage, hydroformage et fabrication additive. ISBN : 978-1-78405-194-5, 262 pages, éditions ISTE - Février 2017.

[REE 99] D.W.A. Rees. «Instability theory for ellipsoidal bulging of rolled sheet. » Journal of Materials Processing Technology, 92-93:508-517, 1999.

[STO 83] B. Storakers, "Finite plastic deformation of a circular membrane under hydrostatic pressure. » Int. J. Mech. Sci., 8 :619-628, 1983. 
[YUA 06] S. J. Yuan, C. Han, and X. S. Wang, « Hydroforming of automotive structural components with rectangularsections », International Journal of Machine Tools and Manufacture, 46 :1201-1206, 2006.

[WAN 69] N.M. Wang and M.R. Shammany. " On the plastic bulging of a circular diaphragm by hydrostatic pressure. » Mech. Phys. Solids, 17 :43-59, 1969

[ZHE 03] T. Zheng-Xue and Y. Lin. " Characterization of mechanical properties of thin polymer films using a bi-axial tension based on blow-up test », Plastics, Rubber and composites, 32(10) : 4-59, 2003. 